\title{
A Study on the Performance of Large Bayes Classifier
}

\author{
Dimitris Meretakis, Hongjun Lu, and Beat Wüthrich \\ Computer Science Department, Hong Kong University of Science and Technology, \\ Clear Water Bay, Hong Kong, China \\ \{meretaks, luhj, beat\}@cs.ust.hk
}

\begin{abstract}
Large Bayes (LB) is a recently introduced classifier built from frequent and interesting itemsets. LB uses itemsets to create context-specific probabilistic models of the data and estimate the conditional probability $P\left(c_{i} \mid A\right)$ of each class $c_{i}$ given a case $A$. In this paper we use chi-square tests to address several drawbacks of the originally proposed interestingness metric, namely: (i) the inability to capture certain really interesting patterns, (ii) the need for a userdefined and data dependent interestingness threshold, and (iii) the need to set a minimum support threshold. We also introduce some pruning criteria which allow for a trade-off between complexity and speed on one side and classification accuracy on the other. Our experimental results show that the modified LB outperforms the original LB, Naïve Bayes, C4.5 and TAN.
\end{abstract}

\section{Introduction}

Until recently association (descriptive) and classification (predictive) mining have been considered as disjoint research and application areas. Descriptive mining aims at the discovery of strong local patterns, so-called itemsets [1] that hopefully provide insights on the relationships among some of the attributes of the database. Predictive mining deals with databases that consist of labeled tuples. Each label represents a class and the aim is to discover a model of the data that can be used to determine the labels (classes) of previously unseen cases.

The use of association mining techniques for classification purposes has only recently been explored. Following this route we recently proposed Large Bayes (LB) classifier [5]. LB considers each attribute-value pair as a distinct item and assumes that the training set is a set of transactions. During the learning phase LB employs an Apriori-like [1] association mining algorithm to discover interesting and frequent labeled itemsets. In the context of classification, we define a labeled itemset $l$ as a set of items together with the supports $l_{\text {. }} u_{u} p_{i}$ for each possible class $c_{i}$. In other words a labeled itemset provides the observed probability distribution of the class variable given an assignment of values for the corresponding attributes: $l . \sup _{i}=P\left(l, c_{i}\right)$.

A new case $A=\left\{a_{1} a_{2} \ldots a_{n}\right\}$, is assigned to the class $c_{i}$ with the highest conditional probability $P\left(c_{i} \mid A\right)=P\left(A, c_{i}\right) / P(A)$. Since the denominator is constant with respect to $c_{i}$ it can be ignored and the object is said to be in class $c_{i}$ with the highest value $P\left(A, c_{i}\right)$. LB selects the longest subsets of $A$ that are present in the set of discovered itemsets and uses them to incrementally build a product approximation of $P\left(A, c_{i}\right)$. For example, if $A=\left\{a_{1} a_{2} a_{3} a_{4} a_{5}\right\}$, a valid product approximation would be:

$$
P\left(A, c_{i}\right)=P\left(a_{2} a_{5} c_{i}\right) P\left(a_{3} \mid a_{5} c_{i}\right) P\left(a_{1} \mid a_{2} a_{3} c_{i}\right) P\left(a_{4} \mid a_{1} a_{5} c_{i}\right) .
$$


Fig. 1 illustrates how this product approximation is incrementally generated from the set of longest itemsets by adding one itemset at each step. The formula is subsequently evaluated using the class supports of the selected itemsets and finally the class $c_{i}$ with the highest probability $P\left(l, c_{i}\right)$ is assigned to $A$. Note that this process builds on the fly a local probabilistic model for the approximation of $P\left(A, c_{i}\right)$ that only holds for the particular classification query.

\begin{tabular}{|c|c|c|l|l|}
\hline step & covered items iset selected & \multicolumn{1}{|c|}{ product approximation } & \multicolumn{1}{c|}{ available itemsets } \\
\hline 0 & $\varnothing$ & $\varnothing$ & $\mathrm{N} / \mathrm{A}$ & $\left\{a_{1} a_{2} a_{3}\right\},\left\{a_{1} a_{4} a_{5}\right\},\left\{a_{2} a_{5}\right\},\left\{a_{3} a_{4}\right\},\left\{a_{3} a_{5}\right\}$ \\
\hline 1 & $\left\{a_{2} a_{5}\right\}$ & $\left\{a_{2} a_{5}\right\}$ & $P\left(a_{2} a_{5} c_{1}\right)$ & $\left\{a_{1} a_{2} a_{3}\right\},\left\{a_{1} a_{4} a_{5}\right\},\left\{a_{3} a_{4}\right\},\left\{a_{3} a_{5}\right\}$ \\
\hline 2 & $\left\{a_{2} a_{3} a_{5}\right\}$ & $\left\{a_{3} a_{5}\right\}$ & $P\left(a_{2} a_{5} c_{i}\right) P\left(a_{3} \mid a_{5} c_{I}\right)$ & $\left\{a_{1} a_{2} a_{3}\right\},\left\{a_{1} a_{4} a_{5}\right\},\left\{a_{3} a_{4}\right\}$ \\
\hline 3 & $\left\{a_{1} a_{2} a_{3} a_{5}\right\}$ & $\left\{a_{1} a_{2} a_{3}\right\}$ & $P\left(a_{2} a_{5} c_{i}\right) P\left(a_{3} \mid a_{5} c_{I}\right) P\left(a_{1} \mid a_{2} a_{3} c_{I}\right)$ & $\left\{a_{1} a_{4} a_{5}\right\},\left\{a_{3} a_{4}\right\}$ \\
\hline 4 & $\left\{a_{1} a_{2} a_{3} a_{4} a_{5}\right\}$ & $\left\{a_{1} a_{4} a_{5}\right\}$ & $P\left(a_{2} a_{5} c_{i}\right) P\left(a_{3} \mid a_{5} c_{I}\right) P\left(a_{1} \mid a_{2} a_{3} c_{I}\right) P\left(a_{4} \mid a_{1} a_{5} c_{i}\right)$ & $\left\{a_{3} a_{4}\right\}$ \\
\hline
\end{tabular}

Fig. 1. Incremental construction of a product approximation for $P\left(a_{1} a_{2} a_{3} a_{4} a_{5} c_{i}\right)$

The key factor in this process is the selection of interesting itemsets. In [5] we used an interestingness metric that was an adaptation of the well known cross-entropy between two probability distributions. To overcome the drawbacks of this approach we use chi-square $\left(\chi^{2}\right)$ tests to identify interesting itemsets. In section 4 we show experimentally that this approach leads to significant performance improvements. Moreover, we deal with the problem of setting the correct minimum support and interestingness thresholds for each data set. Although the settings we suggested in [5] work relatively well in practice, they are empirically determined and lack intuitive justification. The $\chi^{2}$ test besides stemming directly from statistical theory also provides intuitive interpretation to the thresholds.

We also discuss the effect of two other pruning criteria on the performance of the classifier, namely pruning based on (a) the support and (b) the conditional entropy of the class given an itemset. Use of these criteria often leads to the generation of smaller classifiers often without significant sacrifice in the classification accuracy.

\section{An Overview of Large Bayes Classifier}

We will briefly outline the original LB algorithm, which is described in more details in [5]. Large Bayes is a classifier build from labeled itemsets, denoted as itemsets in the sequel. Consider a domain where instances are represented as instantiations of a vector $\boldsymbol{A}=\left\{A_{1}, A_{2}, \ldots, A_{n}\right\}$ of $n$ discrete variables, where each variable $A_{i}$ takes values from $\operatorname{val}\left(A_{i}\right)$ and each instance is labeled with one of the $|\operatorname{val}(C)|$ possible class labels, where $C$ is the class-variable. A labeled itemset $l$ with its class supports l.sup provides the probabilities of joint occurrence $P\left(l, c_{i}\right)$ for $l$ and each class $c_{i}$. The learning phase of Large Bayes aims to discover such itemsets that are frequent and interesting. As usual, an itemset is frequent, if its support is above the user defined minimum support threshold minsup: $\frac{1}{|D|} \cdot \sum_{i=1 . . . \text { val }(c) \mid} l_{\text {count }} \geq$ minsup $_{\text {. }}$

We can derive an estimation of the class-supports of an itemset $l$ using two subsets of 1 where one item is missing. Consider for example the itemset $l=\left\{a_{1}, a_{2}, a_{3}\right)$. Its class-supports l.sup $_{i}=P\left(l, c_{i}\right)=P\left(a_{1}, a_{2}, a_{3}, c_{i}\right)$ can be estimated using $l_{1}=\left\{a_{1}, a_{2}\right\}$ and $l_{2}$ 
$=\left\{a_{1}, a_{3}\right\}$ by implicitly making certain independence assumptions: $P\left(a_{1}, a_{2}, a_{3}, c_{i}\right)=$ $P\left(a_{1}, a_{2}, c_{i}\right) P\left(a_{3} \mid a_{1}, c_{i}\right)=P\left(l_{1}, c_{i}\right) P\left(l_{2}, c_{i}\right) / P\left(l_{1} \cap l_{2}, c_{i}\right)$. Roughly speaking, if this estimation is accurate then $l$ itself is not interesting, since it does not provide any more information than its subsets $l_{1}$ and $l_{2}$. The quality of the approximation is quantified with an interestingness measure $I(l)$ that returns zero if $P\left(l, c_{i}\right)$ is actually equal to $P\left(l_{1}, c_{i}\right) P\left(l_{2}, c_{i}\right) / P\left(l_{1} \cap l_{2}, c_{i}\right)$ and increases with their difference. An itemset is interesting if $I(l)>\tau$, where $\tau$ is a user-defined threshold. Fig. 2 presents the learning phase, which performs an Apriori-like bottom-up search and discovers the set $F$ of itemsets that will be used to classify new cases.

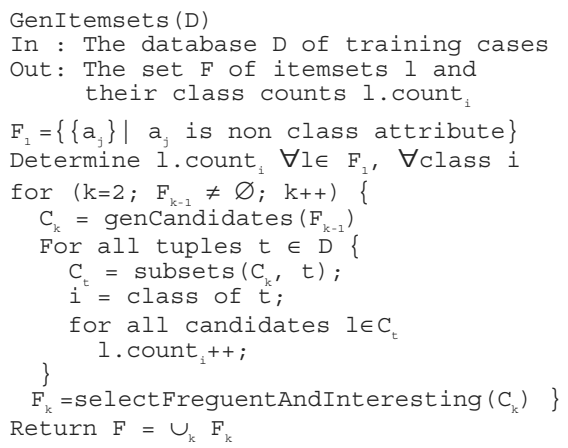

Fig. 2. Algorithm genItemsets

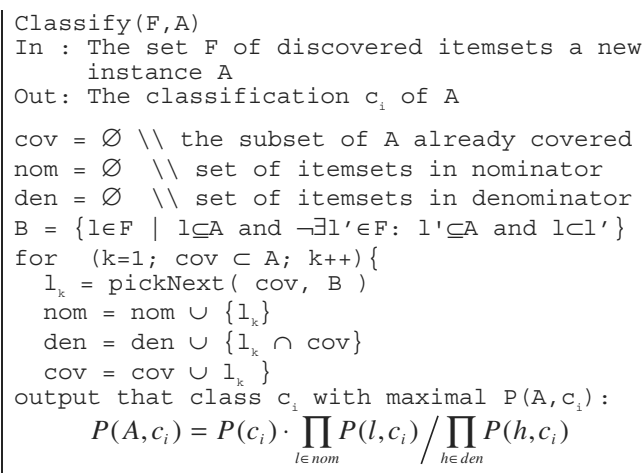

Fig. 3. Algorithm classify

Given a particular instance $A$ to be classified, the set $F^{\prime}$ of the longest and most interesting itemsets in $F$ which are subsets of $A$ are selected. The itemsets of $F^{\prime}$ are then used to incrementally construct a product approximation for $P\left(A, c_{i}\right)$. The procedure classify() that performs this task is presented in Fig. 3 while Fig. 4 presents the selection criteria for the next itemset to be inserted in the product approximation.

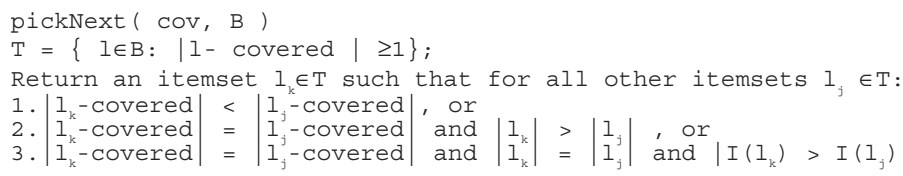

Fig. 4. Procedure pickNext

The resulting formula is the local model build on the fly by LB to classify $A$. This model implies some conditional independence assumptions among the variables but they are context-specific in the sense that different classification queries (i.e. different values of $A$ ) will produce different models making different independence assumptions. [5,6] discuss this in more detail. Finally, the formula $P\left(A, c_{i}\right)$ is evaluated for each $c_{i}$ and $A$ is labeled with the class $c_{i}$ that maximizes $P\left(A, c_{i}\right)$.

\section{Improving Large Bayes}

A key factor affecting the performance of Large Bayes is the accurate identification of interesting itemsets. The interestingness of an itemset $l$ is defined in terms of the error 
when estimating $P\left(l, c_{i}\right)$ using subsets of $l$. Let $l$ be an itemset of size $|l|$ and $l_{j}, l_{k}$ be two $(|l|-1)$-itemsets obtained from $l$ by omitting the $j^{\text {th }}$ and $k^{\text {th }}$ item respectively. We can use $l_{j}, l_{k}$ to produce an estimate $P_{j, k}\left(l, c_{i}\right)$ of $P\left(l, c_{i}\right)$ :

$$
P_{e s t}\left(l, c_{i}\right)=P_{j, k}\left(l, c_{i}\right)=\frac{P\left(l_{j}, c_{i}\right) \cdot P\left(l_{k}, c_{i}\right)}{P\left(l_{j} \cap l_{k}, c_{i}\right)}
$$

Our goal is to keep those itemsets only, for which the corresponding observed probabilities differ much from the estimated ones. Information-theoretic metrics such as the cross-entropy (or Kullback-Leibler distance) are widely used [4] as a measure of the distance between the observed and the estimated probability distributions:

$$
D_{K L}\left(P, P_{e s t}\right)=\sum_{l, c_{i}} P\left(l, c_{i}\right) \log \frac{P\left(l, c_{i}\right)}{P_{e s t}\left(l, c_{i}\right)}
$$

In our case, however, the goal is to measure the distance between specific elements of the probability distribution. Consider for example a case with two variables $A_{1}$ and $A_{2}$ and $\left|\operatorname{val}\left(A_{1}\right)\right|=\left|\operatorname{val}\left(A_{2}\right)\right|=4$. The corresponding sixteen 2 -itemsets define the complete observed joint probability distribution $P\left(A_{1}, A_{2}, C\right)$. A high value of such metrics suggests that the class-supports of the corresponding itemsets cannot be accurately approximated on average by the class-supports of their subsets. To measure the accuracy of the approximation for individual itemsets in [5] we defined the interestingness $I\left(l \mid l_{j}, l_{k}\right)$ of $l$ with respect to its subsets $l_{j}$ and $l_{k}$ as:

$$
I\left(l \mid l_{j}, l_{k}\right)=\sum_{c_{i}}\left|P\left(l, c_{i}\right) \log \frac{P\left(l, c_{i}\right)}{P_{e s t}\left(l, c_{i}\right)}\right|
$$

This ad-hoc measure presents certain drawbacks with respect to its ability to identify interesting local patterns. Consider for example a domain with two classes and an itemset $l$ for which $P\left(l, c_{1}\right)=0, P\left(l, c_{2}\right)=0.15$ and $P_{\text {est }}\left(l, c_{1}\right)=0.1, P_{\text {est }}\left(l, c_{2}\right)=0.15$. Although this is indeed a very interesting itemset since the estimated probability for $c_{I}$ greatly differs from observed one, $I\left(l \mid l_{j}, l_{k}\right)=0$ and $l$ is discarded as non-interesting. In addition, our interestingness measure (but also every information-theoretic measure) suffers from the fact that it ignores the sample size and assumes that the sample probability distribution is equal to the population probability distribution thus ignoring the possibility that the differences occurred purely because of chance.

In the sequel we describe the application of chi-square $\left(\chi^{2}\right)$ tests to overcome these problems. We reduce the problem of deciding whether an itemset is interesting to applying a hypothesis-testing procedure on the following hypotheses:

$H_{0}: P\left(l, c_{i}\right)=P_{e s t}\left(l, c_{i}\right)$, i.e. $l$ is not-interesting

$H_{l}: P\left(l, c_{i}\right) \neq P_{\text {est }}\left(l, c_{i}\right)$, i.e. $l$ is interesting

To test the hypotheses we calculate the $\chi^{2}$ test statistic with $|C|$ degrees of freedom. $(|D|$ is the database size, $|\mathrm{C}|$ the number of classes):

$$
\chi_{l}^{2}=\sum_{j=1}^{|C|} \frac{\left(P\left(l, c_{i}\right) \cdot|D|-P_{e s t}\left(l, c_{i}\right) \cdot|D|\right)^{2}}{P_{e s t}\left(l, c_{i}\right) \cdot|D|}=\sum_{j=1}^{|C|} \frac{\left(P\left(l, c_{i}\right)-P_{e s t}\left(l, c_{i}\right)\right)^{2}}{P_{e s t}\left(l, c_{i}\right)} *|D|
$$

If $\chi_{l}^{2}>\chi_{p,|c|}^{2}$ the null hypothesis $H_{0}$ is rejected and $l$ is considered interesting. The statistical-significance threshold $p$ is user-defined but should in general be high i.e. $\mathrm{p}<0.05$ since discovering non-interesting itemsets does not improve the accuracy and unnecessarilly increases the complexity of the resulting classifier. The degrees of freedom for the test are $|C|$ since the sums of the expected and the observed 
frequencies of an itemset are generally different [8]. If the degrees of freedom are two or less, Yates correction is applied (subtracting 0.5 from the absolute difference in Eq. (4) before squaring, when this difference exceeds 0.5 ).

A problem associated with $\chi^{2}$ tests is that the estimated frequencies $P_{e s t}\left(l, c_{i}\right) \cdot|D|$ in each term of in Eq. (4) should be not too small otherwise the test is sensitive to errors. To overcome this problem we apply a merging step before calculating the $\chi^{2}$ statistic. During this step the class with the smallest frequency is merged with the immediate larger class to form a composite class containing the sum of the frequencies. The corresponding observed frequencies are merged also and the degrees of freedom $(d f)$ are reduced by one. The merging phase stops when all expected frequencies are large enough or when all class-frequencies are merged. Following standard statistical practice we set the minimum value of an expected frequency to 5 if $d f=2$ and 3 if $d f>2$, otherwise it is merged.

As a result of the merging step, each itemset $l$ has a value $\chi_{l}^{2}$ that refers to different degrees of freedom $d f_{l}$. To compare these values with the minimum required threshold $\chi_{p,|c|}^{2}$ we need a degrees-of-freedom-independent test. For that reason we take advantage of the fact that the value $t=\sqrt{2 \cdot \chi^{2}}-\sqrt{2 \cdot d f-1}$ approximately follows the normal distribution and therefore the modified requirement for an itemset to be interesting becomes:

$$
t=\sqrt{2 \cdot \chi_{l}^{2}}-\sqrt{2 \cdot d f_{l}-1}>\sqrt{2 \cdot \chi_{p,|C|}^{2}}-\sqrt{2 \cdot|C|-1}
$$

Note that although $t$ can now take negative values as well, the requirement for an itemset to be interesting remains that its $t$ value is bigger than the threshold of equation (5) which is determined by the required statistical significance level $p$ and the number of classes $|C|$.

\subsection{Pruning Criteria: Trading off Accuracy for Simplicity and Speed}

A difficult challenge in the design of classifiers is preventing overfitting and generating simple models. Simple models are not only easily interpretable but also generalize better in unseen data and are faster to build and evaluate. In LB overfitting translates to the discovery of "too many itemsets" and this is particularly true in domains with many multi-valued attributes, where the search space is huge. $\chi^{2}$ tests significantly reduce the number of discovered itemsets to a tractable amount. However, there are some other prunning criteria that can potentially reduce the number of itemsets and accelerate both the learning and classification phase.

Support-based pruning is used by many classification methods including decision trees, where a leaf is not expanded if it contains less than a minimum number of cases. In section 4 we evaluate the effect of support pruning on the accuracy of LB.

A somehow more effective pruning criterion is the conditional entropy of the class $C$ given an itemset $l$ :

Conditional entropy

$$
H(C \mid l)==\sum_{j=1}^{|c|} P\left(c_{i} \mid l\right) \cdot \log P\left(c_{i} \mid l\right)
$$

zero (if $l$ only appears with a single class) to $\log (|C|)$, if $l$ 's appearances are uniformly distributed among the classes. If $H(C \mid l)$ is very small $l$ bears almost certainty about a class and therefore needs not be expanded. In the next section we show that the 
introduction of a relatively low conditional entropy threshold often reduces the size of the classifier without significantly affecting its accuracy.

\section{Experimental Results}

To evaluate the performance of LB with the $\chi^{2}$ tests (LB-chi2), we use 23 data sets from the UCI ML Repository 77] with a special preference on the largest and more challenging ones in terms of achievable classification accuracy. We compared LBchi2 with the originally proposed version of LB, the Naïve Bayes classifier [2] (since in the extreme case if only 1-itemsets are used LB reduces to NB), Quinlan's Decision Tree classifier C4.5 [9], and TAN [4]; a Bayesian Network classifier that relaxes the independence assumptions of NB by using some pairs of attributes.

Accuracy was measured either using 10-fold cross validation (CV-10) for small data sets or the holdout method (training and testing set split) for the larger ones. The train and test set splits and the cv-folds were the same for all results reported. Since all methods except of C4.5 only deal with discrete attributes, we used entropy-based [3] discretization for all continuous attributes. No discretization was applied for C4.5.

The factor most affecting the results is the p-value of the $\chi^{2}$ tests. We experimented with $0.01,0.025,0.005,0.001$ and 0.0005 , and selected 0.005 as the most effective one. Higher p-values slowly deteriorated the accuracy and tended to produce more complex, larger and slower classifiers. This is natural since high p-values cause more itemsets to be characterized as interesting. On the other hand, values below $p=0.005$ caused most of the itemsets to be rejected as non-interesting and generated simplistic classifiers with poor accuracy. The effects of the varying p-values on the average accuracy and classifier size can be seen on figure 5 .

Table 1 provides a comparison of the algorithms in the 23 data sets according to five criteria. LB-chi2 outperforms all others according to all criteria indicating that it is indeed a very accurate classifier. The criteria used are: (1) Average Accuracy of the classifiers, (2) Average Rank (Smallest values indicate better performance on average), (3) The number of wins-losses of LB-chi2 against other algorithms, and the statistical significance of the improvement of LB-chi2 against each algorithm using (4) a one-sided paired t-test and (5) a Wilcoxon paired, signed, one-sided, rank test.

Table 1. Comparison of the classifiers according to various criteria

$\begin{array}{ccccccc} & & \text { NB } & \mathbf{C 4 . 5} & \text { TAN } & \text { LB } & \text { LB-chi2 } \\ 1 & \text { Average Accuracy } & 0.8187 & 0.8147 & 0.8376 & 0.8332 & 0.8434 \\ 2 & \text { Average Rank } & 3.695652 & 3.73913 & 2.73913 & 2.652174 & 1.913043 \\ 3 & \text { No wins vs.: } & 19-4 & 19-4 & 16-6 & 15-7 & - \\ 4 & \text { 1-side Paired t-test } & 0.9995 & 0.9991 & 0.9940 & 0.9828 & -- \\ 5 & \text { Wilcoxon paired signed rank test } & >0.995 & >0.995 & >0.99 & >0.975 & --\end{array}$


Table 2. Summary Table of datasets and results. $|\mathrm{A}|=$ number of attributes, $|\mathrm{I}|=$ number of distinct items (attribute-value pairs) after discretization, $|\mathrm{C}|=$ number of classes, Miss = presence of missing values. Last two colums indicate the training/testing time of LB-chi2 in sec

\begin{tabular}{|c|c|c|c|c|c|c|c|c|c|c|c|c|c|c|}
\hline \multirow{2}{*}{\multicolumn{2}{|c|}{ Data Set }} & \multicolumn{6}{|c|}{ Data set Properties } & \multicolumn{5}{|c|}{ Accuracy } & \multicolumn{2}{|c|}{ Time (s) LB-chi2 } \\
\hline & & $|\mathrm{A}|$ & $|\mathrm{I}|$ & $|\mathrm{C}|$ & Mis $\neq$ & \# Train & \# Test & NB & C4.5 & TAN & LB & LB-chi2 & Train & Test \\
\hline 1 & Adult & 14 & 147 & 2 & Yes & 32561 & 16281 & 0.8412 & 0.854 & 0.8571 & 0.8511 & 0.8668 & 48.81 & 37.04 \\
\hline 2 & Australian & 14 & 48 & 2 & No & 690 & CV -10 & 0.8565 & 0.8428 & 0.8522 & 0.8565 & 0.8609 & 0.18 & 0.03 \\
\hline 3 & Breast & 10 & 28 & 2 & Yes & 699 & $\mathrm{CV}-10$ & 0.97 & 0.9542 & 0.9671 & 0.9686 & 0.9714 & 0.11 & 0.02 \\
\hline 4 & Chess & 36 & 73 & 2 & No & 2130 & 1066 & 0.8715 & 0.995 & 0.9212 & 0.9024 & 0.9418 & 1.99 & 2.20 \\
\hline 5 & Cleve & 13 & 27 & 2 & Yes & 303 & CV-10 & 0.8278 & 0.7229 & 0.8122 & 0.8219 & 0.8255 & 0.07 & 0.01 \\
\hline 6 & Flare & 10 & 27 & 2 & No & 1066 & $\mathrm{CV}-10$ & 0.79 & 0.8116 & 0.8264 & 0.8152 & 0.818 & 0.18 & 0.03 \\
\hline 7 & German & 20 & 60 & 2 & No & 999 & $\mathrm{CV}-10$ & 0.741 & 0.717 & 0.727 & 0.748 & 0.75 & 0.42 & 0.07 \\
\hline 8 & Heart & 13 & 17 & 2 & No & 270 & $\mathrm{CV}-10$ & 0.8222 & 0.7669 & 0.8333 & 0.8222 & 0.8185 & 0.05 & 0.01 \\
\hline 9 & Hepatitis & 19 & 32 & 2 & Yes & 155 & $\mathrm{CV}-10$ & 0.8392 & 0.8 & 0.8188 & 0.845 & 0.8446 & 0.05 & 0.01 \\
\hline 0 & Letter & 16 & 146 & 26 & No & 15000 & 5000 & 0.7494 & 0.777 & 0.8572 & 0.764 & 0.8594 & 109.29 & 56.90 \\
\hline 1 & Lymph & 18 & 49 & 4 & No & 148 & $\mathrm{CV}-10$ & & & & 0.8457 & 0.8524 & 0.07 & 0.02 \\
\hline 2 & Pendigits & 16 & 151 & 10 & No & 7494 & 3499 & 0.8350 & 0.923 & 0.9360 & 0.9182 & 0.9403 & 44.23 & 22.58 \\
\hline 3 & Pima & 8 & 15 & 2 & No & 768 & CV-10 & 0.759 & 0.711 & 0.7577 & 0.7577 & 0.7564 & 0.06 & 0.02 \\
\hline 4 & $\begin{array}{c}\text { Pima } \\
\text { Diabetes }\end{array}$ & 8 & 14 & 2 & No & 768 & $\mathrm{CV}-10$ & 0.7513 & 0 . & 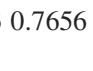 & 669 & 0.763 & 0.07 & 0.02 \\
\hline 5 & Satimage & 36 & 384 & 6 & No & 4435 & 2000 & 0.818 & 0.852 & 0.872 & 0.839 & 0.8785 & 392.60 & 83.75 \\
\hline 6 & Segment & 19 & 147 & 7 & No & 1540 & 770 & 0.9182 & 0.958 & 0.9351 & 0.9416 & 0.9429 & 2.28 & 1.16 \\
\hline 7 & $\begin{array}{l}\text { Shuttle- } \\
\text { small }\end{array}$ & 9 & 50 & 7 & No & 3866 & 1934 & 0.987 & 0.995 & 0.9964 & 0.9938 & 0.9948 & 1.40 & 0.78 \\
\hline 8 & Sleep & 13 & 113 & 6 & No & 70606 & 35305 & 0.6781 & 0.7310 & 0.7306 & 0.7195 & 0.7353 & 476.71 & 621.97 \\
\hline 9 & Splice & 59 & 287 & 3 & No & 2126 & 1064 & 0.9464 & 0.933 & 0.9463 & 0.9464 & 0.9408 & 3.24 & 3.07 \\
\hline 0 & Vehicle & 18 & 69 & 4 & No & 846 & $\mathrm{CV}-10$ & 0.6112 & 0.6982 & 0.7092 & 0.688 & 0.7187 & 1.24 & 0.23 \\
\hline & $\begin{array}{c}\text { Vote } \\
\text { Records }\end{array}$ & 16 & 48 & 2 & No & 435 & $C \mathrm{CV}, \mathrm{l}$ & 0.9034 & 0.9566 & 0.9332 & 0.9472 & .9334 & 0.13 & 0.04 \\
\hline & $\begin{array}{l}\text { Vaveform- } \\
21\end{array}$ & 21 & 44 & 3 & No & 300 & 4700 & 0.7851 & 0.704 & 0.7913 & 0.7943 & 0.7913 & 0.1 & 2.724 \\
\hline & Yeast & 8 & 18 & 10 & No & 1484 & $C v-14$ & 0.5805 & 0.5573 & 0.5721 & 0.5816 & 0.5816 & 0.15 & 0.04 \\
\hline
\end{tabular}

Table 2 provides information about the data sets, lists the accuracies of the classifiers and shows the training and testing time of LB-chi2 on all data sets (Measured on a 400MHz Pentium WinNT PC). Noticeably, the biggest improvements in accuracy against the original LB came mostly from the largest data sets; this indicates the inability of the originally used interestingness metric in such cases.

The p-value for chi2-LB was set to 0.005 and to facilitate more accurate $\chi^{2}$ tests the minimum support was set to $\max \{10,2 *|c|\}$. Although this is a minimum requirement in order for the test statistic to be accurate this can be further increased in order to reduce both the training time and the size of the classifier as discussed below. 

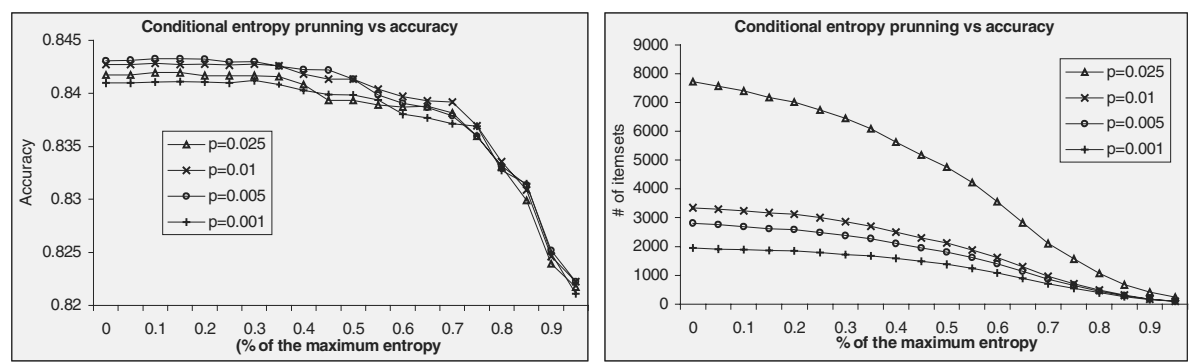

Fig. 5. Effect of conditional entropy pruning on (a) accuracy and (b) size of classifier for four different $\mathrm{p}$-value thresholds. $\mathrm{x}$-axis contains the threshold as a percentage of the maximum conditional entropy $\log |C|$

Figure 5 illustrates the effect of conditional entropy pruning on the average accuracy (5a) and size (5b) of LB. Since the number of classes is diferent among the datasets the minimum threshold $\min H$ is expressed as a percentage of the maximum conditional entropy $\log |C|$. In a 4-class domain, for example, a value of 0.2 implies that $\min H=0.2 \cdot \log 4=0.4$. Values for $\min H$ of up to $0.3 \cdot \log |C|$ have little impact on the accuracy while at the same time reducing the size of the classifier. The rightmost values of the graph correspond to maximum pruning where only 1-itemsets are used and therefore represent the accuracy and size of Naïve Bayes classifier.

Support pruning has a more drastic effect on the size of the classifier as can be seen in Figure 6. This is particularly true on large data sets like "sleep" where 10 occurrences for an itemset $l$ represent a probability $P(l)=0.0001$. Increasing the minsup threshold to 0.005 in this data set reduced the number of itemsets discovered from 31000 to 7500 while the accuracy fell only slightly, from 0.7336 to 0.727 .

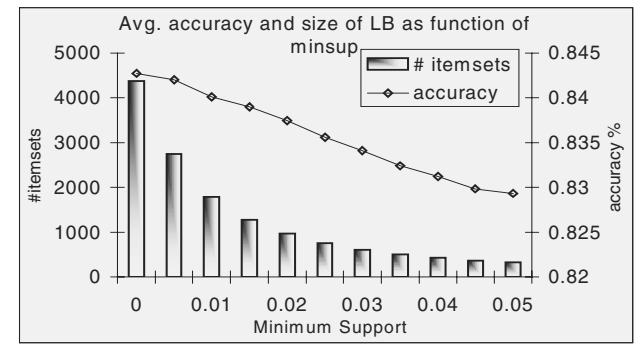

Fig. 6. Effect of support pruning on average accuracy and size of LB

\section{References}

1. R. Agrawal, R. Srikant, Fast algorithms for mining association rules, VLDB-94, 1994.

2. R.Duda, P.Hart, Pattern Classification and Scene Analysis, John Wiley \& Sons, 1973.

3. U.M.Fayyad, K.B.Irani, Multi-Interval discretization of continuous-valued attributes for classification learning, 13th IJCAI, 1022-1027, 1993. 
4. N.Friedman, D. Geiger, M. Goldszmidt, Bayesian Network Classifiers, Machine Learning, 29, 131-163, 1997.

5. D. Meretakis, B.Wüthrich, Extending Naïve Bayes Classifiers Using Long Itemsets, KDD99, pp 165-174, San Diego, USA, 1999.

6. D.Meretakis, B.Wüthrich, Classification as Mining and Use of Labeled Itemsets, ACM SIGMOD Workshop on Research Issues in Data Mining and Knowledge Discovery (DMKD'99), Philadelphia, USA, 1999.

7. C.J.Merz, P.Murphy, UCI repository of machine learning databases, 1996 (http:://www.cs.uci.edu/ mlearn/MLRepository.html).

8. W.H. Press, S.A. Teukolsky, W.T. Vetterling, B.P. Flannery, Numerical Recipes in C, 2nd Ed, Cambridge University Press, 1992.

9. J.R.Quinlan, C4.5: Programs for Machine Learning, Morgan Kaufmann, 1993. 